\title{
formation through modulation of IRS1-AKT glucose uptake and HSP-elicited UPR in lung cancer
}

Chantal Hoi Yin Cheung ${ }^{1}$, Chia-Lang Hsu ${ }^{1,2}$, Tsai-Yu Lin', Wei-Ting Chen', Yi-Ching Wang ${ }^{3,4}$, Hsuan-Cheng Huang ${ }^{5^{*}}$ and Hsueh-Fen Juan ${ }^{1,6^{*}}$ (D)

\begin{abstract}
Background: ZNF322A is an oncogenic transcription factor that belongs to the Cys2His2-type zinc-finger protein family. Accumulating evidence suggests that ZNF322A may contribute to the tumorigenesis of lung cancer, however, the ZNF322A-mediated downstream signaling pathways remain unknown.

Methods: To uncover ZNF322A-mediated functional network, we applied phosphopeptide enrichment and isobaric labeling strategies with mass spectrometry-based proteomics using A549 lung cancer cells, and analyzed the differentially expressed proteins of phosphoproteomic and proteomic profiles to determine ZNF322A-modulated pathways.

Results: ZNF322A highlighted a previously unidentified insulin signaling, heat stress, and signal attenuation at the posttranslational level. Consistently, protein-phosphoprotein-kinase interaction network analysis revealed phosphorylation of IRS1 and HSP27 were altered upon ZNF322A-silenced lung cancer cells. Thus, we further investigated the molecular regulation of ZNF322A, and found the inhibitory transcriptional regulation of ZNF322A on PIM3, which was able to phosphorylate IRS1 at serine ${ }^{1101}$ in order to manipulate glucose uptake via the PI3K/AKT/mTOR signaling pathway. Moreover, ZNF322A also affects the unfolded protein response by phosphorylation of $\mathrm{HSP}_{2} 7^{\mathrm{S} 2}$ and elF2a ${ }^{\mathrm{SS1}}$, and triggers autophagosome formation in lung cancer cells.
\end{abstract}

Conclusions: These findings not only give new information about the molecular regulation of the cellular proteins through ZNF322A at the post-translational level, but also provides a resource for the study of lung cancer therapy.

Keywords: Autophagy, Glucose starvation, Heat stress, Lung cancer, Phosphoproteomics, Proteomics, Zinc-finger protein

\footnotetext{
* Correspondence: hsuancheng@ym.edu.tw; yukijuan@ntu.edu.tw

${ }^{5}$ Institute of Biomedical Informatics, National Yang-Ming University, No.155,

Sec.2, Linong Street, Taipei 11221, Taiwan

'Department of Life Science, National Taiwan University, Taipei 10617,

Taiwan

Full list of author information is available at the end of the article
}

C C The Author(s). 2020 Open Access This article is licensed under a Creative Commons Attribution 4.0 International License, which permits use, sharing, adaptation, distribution and reproduction in any medium or format, as long as you give appropriate credit to the original author(s) and the source, provide a link to the Creative Commons licence, and indicate if changes were made. The images or other third party material in this article are included in the article's Creative Commons licence, unless indicated otherwise in a credit line to the material. If material is not included in the article's Creative Commons licence and your intended use is not permitted by statutory regulation or exceeds the permitted use, you will need to obtain permission directly from the copyright holder. To view a copy of this licence, visit http://creativecommons.org/licenses/by/4.0/ The Creative Commons Public Domain Dedication waiver (http://creativecommons.org/publicdomain/zero/1.0/) applies to the data made available in this article, unless otherwise stated in a credit line to the data. 


\section{Background}

Cys2His2 (C2H2)-type zinc finger (ZNF) is one of the most common DNA-binding motifs and plays a major role as transcription factor, which is encoded by $2 \%$ of human genes [1]. Cumulative evidence shows that the ZNFs fulfill a variety of regulatory roles in cellular processes, including differentiation, development, metabolism, cell survival, apoptosis, and autophagy [2-6]. Besides, C2H2-ZNFs have been reported as either activator or suppressor, depending on the cellular context and target genes. These findings suggest that $\mathrm{C} 2 \mathrm{H} 2-\mathrm{ZNFs}$ are pivotal regulators in tumorigenesis. In our previous study, ZNF322A, a protein belongs to $\mathrm{C} 2 \mathrm{H} 2-\mathrm{ZNF}$ family, contributes to cell proliferation, motility, tumor growth, metabolism, stemness properties, and metastasis [7-9]. Mechanistically, ZNF322A activates the transcriptional activity of serum response element and activator protein-1, and cooperates with c-Jun to activate alpha-adducin and cyclin D1 [7]. Clinically, ZNF322A was found significantly amplified in Asian and Caucasian lung cancer patients with poor prognosis [10]. Our previous quantitative proteomic analysis of ZNF322A-silenced lung cancer cells revealed that the downstream were participated in signal transduction and protein phosphorylation, vesicle-mediated transport, generation of energy and chromatin organization [7]. These findings indicate that ZNF322A plays an important role in lung cancer development, however, the regulatory role of ZNF322A at the phosphorylation level is still unknown.

Cancer is a set of diseases characterized by abnormal alterations that leads to unrestricted cell growth, enhanced metabolism, and suppression of programmed cell death. Dysregulation of kinases and phosphatases is commonly associated with various cancers, thereby conferring the cancer cells with proliferation advantages [11, 12]. Protein phosphorylation is one of the most widespread modes of post-transcriptional modification (PTM) in cell signaling. Many protein kinases such as mitogen-activated protein kinases (MAPKs) and the serine/threonine protein kinase Akt (AKT) are major enzymes that relay signals in response to oncogenic stimuli and drive transcriptional programs in favor of tumor growth $[13,14]$. Therefore, characterization of protein phosphorylation induced signaling changes may provide insights into the regulation of molecular mechanisms involved in tumor progression. Quantitative phosphoproteomic profiling allows researchers to study the aberrant regulation of signaling pathways and also assists in the discovery of appropriate therapeutic targets for diseases [15-17]. With the development of phosphopeptide enrichment strategy and quantitative mass spectrometry-based proteomic analysis, it is possible to profile site-specific phosphorylation events and investigate aberrantly activated signaling pathways in cancer.

Evidence has shown that oncogenic activation of mammalian target of rapamycin (mTOR) signaling induces cellular processes required for cancer cell growth and survival [18]. Among them, the phosphatidylinositol 3kinase/protein kinase $\mathrm{B} /$ mammalian target of rapamycin (PI3K/AKT/mTOR) and MAPK signaling pathways are crucial for autophagosome formation in cancer cells. PI3K/ AKT signaling plays critical roles in cellular physiology and processes, such as glucose homeostasis, lipid metabolism, and cell proliferation. Interestingly, knockdown of AKT reduce insulin-induced glucose uptake by regulating glucose transport through the PI3K/AKT signaling pathway [19]. Autophagy is triggered during nutrient deprivation in cells as a homeostatic response to stress [20]. During autophagosome formation, phagosome converts to autophagosome by autophagic stimulus and stress, and its maturation is completed upon fusion with lysosome [21-24]. Endoplasmic reticulum (ER) is a dynamic organelle that responses to environmental stress through a series of signaling cascades known as the unfolded protein response (UPR). Alteration of ER homeostasis causes accumulation of misfolded or unfolded proteins in the ER, which leads to the ER stress and UPR activation. Recent researches have demonstrated that the activation of the PI3K/AKT signaling pathway occurs in $90 \%$ of non-small cell lung cancer (NSCLC) cell lines, and the inhibition of this signaling cascade is not only important for induction of autophagic cell death but also provides insight for developing new treatments $[25,26]$.

\section{Methods}

\section{Cell culture}

Human lung carcinoma A549 cells (CCL-185) and H1299 cells (CRL-5803) were obtained from the American Type Culture Collection (Manassas, VA, USA). Cells were maintained in Dulbecco's Modified Eagle's Medium (DMEM, Gibco Laboratories, Grand Island, NY, USA) with 10\% fetal bovine serum (Biological Industries, Kibbutz Beit Haemek, Israel) at $37{ }^{\circ} \mathrm{C}$ in a humidified incubator with $5 \% \mathrm{CO}_{2}$.

\section{RNA interference}

A549 cells and H1299 cells were transfected for $48 \mathrm{~h}$ with control (siControl) or ZNF322A (siZNF322A) siRNA by Lipofectamine 3000 (Invitrogen, Waltham, MA, USA) according to the manufacturer's protocol. Transfected cells were harvested at $48 \mathrm{~h}$ post-transfection for further assays (Additional file 1: Fig. S1).

\section{RNA extraction and CDNA synthesis}

Total RNA of A549 cells and H1299 cells was extracted by Direct-zol ${ }^{\mathrm{Tm}}$ RNA MiniPrep (Zymo Research, Irvine, CA, USA) according to the manufacturer's instructions. RNA concentration and quality were determined by NanoDrop ND-1000 UV spectrophotometer (NaniDrop Technologies, Wilmington, DE, USA). $500 \mathrm{ng}$ of total RNA was reverse transcript to cDNA by using RevertAid 
$\mathrm{H}$ Minus First Strand cDNA Synthesis Kit (Thermo Fisher Scientific, Waltham, MA, USA).

\section{Real-time quantitative PCR (qRT-PCR) analysis}

The cDNA samples were amplified and applied by using iQ5 Real-time PCR Detection System (Bio-Rad, Hercules, CA, USA). The mRNA expression levels of the target genes were measured by $\triangle \Delta \mathrm{Ct}$ and normalized to glyceraldehyde3-phosphate dehydrogenase (GAPDH). The qRT-PCR primer sequence were listed in Additional file 2: Table S1.

\section{Protein extraction}

Cells were homogenized on ice using a homogenizer (LABSONIC $^{\bullet} \mathrm{M}$ ultrasonic homogenizer, Sartorius AG, Goettingen, Germany) with $60 \%$ amplitude and 0.6 cycle duration for $2 \mathrm{~min}$ using $12 \mathrm{mM}$ sodium deoxycholate (Sigma-Aldrich, St Louis, MO, USA), $12 \mathrm{mM}$ sodium lauroyl sarcosine (MP Biomedicals, Santa Ana, CA, USA), 50 $\mathrm{mM}$ triethylammonium bicarbonate (Sigma-Aldrich), protease inhibitor cocktail (BioShop, Burlington, Canada), and phosphatase inhibitor cocktail (Bionavas, Toronto, Ontario, Canada). Cell lysate was centrifuged at $16000 \times g$ for $20 \mathrm{~min}$ at $4{ }^{\circ} \mathrm{C}$. Supernatants containing protein extract were then subject to the protein quantification using Pierce $^{\mathrm{mt}}$ BCA Protein Assay Kit (Thermo Fisher Scientific).

\section{Reduction and alkylation}

Protein extract was reduced with $10 \mathrm{mM}$ dithiothreitol (BioShop) at $37^{\circ} \mathrm{C}$ water bath for $30 \mathrm{~min}$, and carboxymethylated with $55 \mathrm{mM}$ iodoacetamide (Sigma-Aldrich) in the dark at room temperature for $30 \mathrm{~min}$. Alkylated proteins were digested with Lys-C $(1: 100 \mathrm{w} / \mathrm{w})$ (WAKO, Osaka, Japan) for $3 \mathrm{~h}$ and then digested with trypsin protease $(1: 100 \mathrm{w} / \mathrm{w})$ (Thermo Fisher Scientific) overnight at $37^{\circ} \mathrm{C}$. The trypsin reaction was inactivated by acidified the peptide solution to a $\mathrm{pH}<3$ using trifluoroacetic acid (Sigma-Aldrich). Then, acidified peptide solution was combined with an equal volume of ethyl acetate (SigmaAldrich) and agitated vigorously for $1 \mathrm{~min}$, followed by centrifugation at $15,700 \times \mathrm{g}$ for $2 \mathrm{~min}$ to separate the aqueous and organic phases. The solution from the aqueous phase was dried using a centrifugal evaporator and then subjected to desalting using Styrenedivinylbenzene Empore disk membranes (SDB-XC) StageTips (\#2340, $3 \mathrm{M}$, Nazarethm, Belgium) and eluted in a buffer containing $0.1 \%(\mathrm{v} / \mathrm{v})$ TFA and $80 \%(\mathrm{v} / \mathrm{v})$ acetonitrile (ACN) [27].

\section{Dimethyl labeling}

$300 \mu \mathrm{g}$ of digested peptide were dried under centrifugal evaporator, then reconstituted in $100 \mathrm{mM}$ TEAB. siRNA Control peptides was labeled with $4 \%(\mathrm{v} / \mathrm{v})$ formaldehydeH2 (Sigma-Aldrich), and siRNA ZNF322A peptides was labeled with $4 \%(\mathrm{v} / \mathrm{v})$ formaldehyde-D2 (Sigma-Aldrich).
$12 \mu \mathrm{L}$ of $0.6 \mathrm{M}$ sodium cyanoborohydride (Sigma-Aldrich) was added to each sample and incubated for $1 \mathrm{~h}$ at room temperature. Dimethyl labeling was inactivated by adding $48 \mu \mathrm{L}$ of $0.1 \%(\mathrm{v} / \mathrm{v})$ ammonium hydroxide (WAKO) on ice, and acidified by adding $30 \mu \mathrm{L}$ of $10 \%$ (v/v) formic acid. The H2-labeled control was combined with the D2 labeled ZNF322A-silenced samples at 1:1 ratio.

\section{Phosphopeptide enrichment and fractionation}

The phosphopeptide was enriched by aliphatic hydroxy acid-modified metal oxide chromatography (HAMMOC), where the home-made lactic acid-modified titania MOC tips were prepared [28]. The lactic acid-modified titania MOC tips were first equilibrated with Solution B $(0.1 \%$ TFA, $80 \% \mathrm{ACN}$ ), and $300 \mathrm{mg} / \mathrm{mL}$ lactic acid. $100 \mu \mathrm{g}$ of desalted peptide mixture was mixed with an equal volume of solution B and $300 \mathrm{mg} / \mathrm{mL}$ lactic acid, and loaded onto the lactic acid-modified titania MOC tips, washed with solution A (0.1\% TFA and 5\% ACN) followed by solution B. Phosphopeptides were eluted with 0.5 and $5 \%$ piperidine (Sigma-Aldrich), and the eluates were immediately acidified with 10\% TFA and 20\% phosphoric acid. For strong cation exchange peptide fractionation, phosphopeptides were eluted sequentially with buffers containing $0.1 \%$ TFA, $5 \% \mathrm{ACN}$, and in an ammonium acetate (WAKO) series $[0,20,50,100$, or $500 \mathrm{mM}]$. The collected eluent were acidified with TFA and desalted. The samples were vacuum-dried, and then the phosphopeptides were resuspended in $0.5 \%$ TFA and analyzed by nanoLC-MS/MS.

\section{NanoLC-MS/MS analysis}

NanoLC-MS/MS analysis was performed on a nanoACQUITY UPLC system (Waters, Milford, MA, USA) connected to an LTQ-Orbitrap XL hybrid mass spectrometer (Thermo Electron, Bremen, Germany) equipped with a nanospray interface (Proxeon, Odense, Denmark). Peptide samples were loaded onto a $2 \mathrm{~cm} \times 180 \mu \mathrm{m}$ capillary trap column and then separated in a $75 \mu \mathrm{m} \times 25 \mathrm{~cm}$ nanoACQUITY $1.7 \mu \mathrm{m}$ BEH C18 column (Waters) at a flow rate of $300 \mathrm{~nL} / \mathrm{min}$. Mobile phase A consisted of $0.1 \%$ formic acid, and solution B consisted of $0.1 \%$ formic acid and $80 \%$ ACN. A linear gradient of 10 to $40 \%$ solution B in $90 \mathrm{~min}$ and 40 to $85 \%$ solution B in 10 min was employed throughout this study. Mass spectra from survey full scans were acquired on the Orbitrap (m/z 350-1500). The resolution was set to 60,000 at $m / z 400$ and the automatic gain control (AGC) was set to $1 \times 10^{6}$ ions. The $\mathrm{m} / z$ values triggering MS/MS were put on an exclusion list for $90 \mathrm{~s}$. The top ten most-intense precursor ions were selected from the MS scan for subsequent collision-induced dissociation MS/MS scan by ion trap (AGC target at 7000). Three biological replicates performed in two technical replicates. 


\section{Data analysis}

Raw MS spectra was processed for peak detection and quantitation by using MaxQuant software version 1.5.2.8 (http://maxquant.org). Peptide identification was performed by using the Andromeda search engine against the Swiss-PROT human database (December 9, 2015, reviewed). Search criteria used in this study were trypsin specificity, fixed modification of carbamidomethyl (C), variable modifications of oxidation $(\mathrm{M})$, phosphorylation (STY), light dimethyl labeling ( $\mathrm{HC} 12 \mathrm{HO})$, and heavy dimethyl labeling (D13CDO), and allowed for up to two missed cleavages [29]. A minimum of seven amino acids in the peptide length was required. The precursor mass tolerance was $3 \mathrm{ppm}$ and the fragment ion tolerance was $0.5 \mathrm{Da}$. By using a decoy database strategy, peptide identification was accepted based on the posterior error probability with a false discovery rate of $1 \%$. Precursor masses of already identified peptides were further searched and recalculated by using the "match between runs" option in MaxQuant. All the spectra and the related information were submitted to ProteomeXChange (http://www.proteomexchange.org/, Project accession PXD015936) and can be inspected by PRIDE Inspector.

\section{Functional and pathway enrichment analysis}

Regulated proteins and phosphoproteins (normalized ratio $\geq 1.5$ or $\leq 0.67$ ) were annotated with GO terms. For the phosphoprotein with multiple phosphoryaltion sites, the largest expression changed of the phosphosite was used for the analysis. Over-represented GO terms were visualized as networks by Cytoscape version 3.2.1 [30]. Each node represents an enriched GO term (corrected $p$-value $<0.05)$, and the size of nodes represents the total number of genes in each GO term. Pie chart in a node represents the proportion of significantly regulated proteins derived from proteome and phosphoproteme (red: protein expression; yellow: protein phosphorylation). The edge represents gene overlap score between nodes over a given threshold (0.5). Pathway analysis was analyzed by REACTOME (v56, March 24, 2016).

\section{Kinase activity map analysis and protein-protein interaction network construction}

Significantly regulated phosphoproteins were used for kinase activity map analysis by using DynaPho software [31] with the $p$-value $<0.05$. The protein-protein interactions of differentially expressed proteins and phosphoproteins as well as predicted kinases were accessed by STRING database (version 10.0), https://string-db.org/, with the highest confidence $(0.9)$. The protein-protein interaction network was depicted by Cytoscape version 3.2.1 [30]. Over-represented motifs were matched to known kinase motifs, and the position weight matrix (PWM) for each over-represented motif was generated based on motif-matching sequences from the results of Motif-X. PWM was constructed by counting the occurrence of each amino acid at each position and normalized at each position by the number of sequences. 300 kinase recognition motifs, resulting in 3656 high-quality kinase-substrate relationships, were obtained from the PhosphoNetworks database.

\section{Immunoblot analysis}

Proteins were separated by $10 \%$ SDS-PAGE and transferred onto a polyvinylidene fluoride (PVDF) membrane (Millipore, Bedford, MA, USA). The membrane was blocked in 5\% non-fat milk/PBST or $5 \%$ bovine serum albumin/PBST for $1 \mathrm{~h}$, then incubated overnight with the following primary antibodies diluted in $5 \%$ non-fat milk/PBST or $5 \%$ bovine serum albumin/PBST at $4{ }^{\circ} \mathrm{C}$ : rabbit anti-ZNF322A (GTX121644, GeneTex, Irvine, CA 92606 USA), mouse anti-actin (MAB1501, Millipore, Billerica, Massachusetts, USA), rabbit anti-HSP27 S82 (MDBio, Taipei, Taiwan), rabbit anti-HSP27 (GTX101145, GeneTex), rabbit antieIF2 $\alpha$ S51 (ab32157, Abcam, Cambridge, UK), rabbit anti-eIF2 $\alpha$ (PA5-27366, Thermo Fisher Scientific), rabbit anti-IRS1 S1101 (A0446, Assay Biotech, Sunnyvale, CA, USA), rabbit anti-IRS1 (\#2382, Cell signaling technology, Danvers, MA, USA), rabbit anti-AKT S473 (\#9271, Cell signaling technology), rabbit anti-AKT (\#9272, Cell signaling technology), rabbit anti-SQSTM1 (GTX100685, GeneTex), rabbit anti-LC3B/MAP1LC3B (NB6001384, Novus Biologicals, CO, USA) and mouse anti-actin (MAB1501, Millipore), rabbit anti-Pim-3 (\#4165, Cell signaling technology), Rabbit anti-mTOR (GTX132803, GeneTex), Rabbit anti-mTOR S2448 (GTX101557, GeneTex). The membrane was then incubated with secondary HRP-conjugated anti-rabbit or antimouse IgG (Sigma-Aldrich) for $2 \mathrm{~h}$ at room temperature. Images were acquired using WesternBright ECL HRP substrate (Advansta, Menlo Park, CA, USA), UVP AutoChemi (UVP, Upland, CA, USA) and FluorChem M (ProteinSimple, San Jose, CA, USA).

\section{Glucose uptake assay}

Glucose uptake was measured using a fluorescent non-metabolizable D-glucose analog 2-[N-(7-nitrobenz-2-oxa-1,3-diazol-4-yl) amino]-2-deoxy-D-glucose (2-NBDG; Cayman, Ann Arbor, Michigan, USA) [32]. $6 \times 10^{4}$ A549 cells were seeded onto glass coverslips in 12-well plate for $24 \mathrm{~h}$, then transfected with control siRNA and ZNF322A siRNA for $48 \mathrm{~h}$. Cells were first pre-incubated in glucose-free and serum-free DMEM (Gibco Laboratories) for $15 \mathrm{~min}$ at $37^{\circ} \mathrm{C}$. Next, cells were incubated in $300 \mu \mathrm{M}$ 2-NBDG for $6 \mathrm{~h}$ at $37^{\circ} \mathrm{C}$ in the absence of glucose, and then washed 3 time with PBS and fixed with $3.7 \%$ paraformaldehyde (Sigma-Aldrich). The coverslips were mounted using 
AntiFade Prolong solution (Molecular Probes, Waltham, MA, USA) overnight. Images were acquired by using fluorescence microscope with Leica HCX FL PLAN 1006/1.25 oil objective, and SPOT Advanced software (Diagnostic Instruments, Sterling Heights, MI, USA). For the quantitative fluorescence microscopy analysis, images were analyzed based on the quantitative guideline in fluorescent microscopy imaging for accuracy and precision quantitation [33]. Randomly selected fields from siControl and siZN322A were analyzed to quantify fluorescence intensity and 2-NBDG molecules using Image J software (ImageJ, U.S. National Institutes of Health, Bethesda, Maryland, USA). The sample size determination was based on the statistical guideline, $(n=50)$ [34].

\section{Transmission electron microscope analysis}

A549 lung cancer cell pellet were fixed in $2.5 \%$ glutaraldehyde in $0.1 \mathrm{M}$ phosphate buffer ( $\mathrm{pH}$ 7.4) overnight at $4{ }^{\circ} \mathrm{C}$. Following three washes in $0.1 \mathrm{M}$ phosphate buffer for $15 \mathrm{~min}$. Sample was post-fixed with $1 \%$ osmium tetraoxide in a $0.1 \mathrm{M}$ phosphate buffer for $90 \mathrm{~min}$. The sample were then embedded in Spurr's resin after dehydration in an acetone series overnight at $60^{\circ} \mathrm{C}$. Sections $(\sim 0.95 \mu \mathrm{m})$ were obtained with a glass knife and stained with $1 \%$ toluidine blue. Ultrasections $(\sim 95 \mathrm{~nm})$ were cut and mounted on nickel or copper grids (75 or 100 mesh with $2.5 \%$ Formvar membrane), and then stained with $2 \%$ uranyl acetate for 20 min and washed. The sections were visualized by transmission electron microscope (H-7650, Hitachi-Science \& Technology, Berkshire, United Kingdom).

\section{Results \\ Quantitative phosphoproteomic profiling of A549 lung cancer cells regulated by ZNF322A}

In our quantitative proteomic study of ZNF322Asilenced A549 lung cancer [7], we revealed that the majority of ZNF322A downstream proteins were involved in signal transduction and protein phosphorylation, thus, the ZNF322A-mediated phosphorylation network was established to better understand the mechanism and biological function of zinc-finger proteins in response to lung cancer. As depicted in Fig. 1a, samples were lysed, labeled with different iTRAQ-tags, then analyzed by LCMS/MS for proteomic profiling in our previous study. For the phosphoproteomic profile, samples were lysed, labeled with stable-isotope dimethyl, phosphopeptide enriched and strong cation exchange (SCX) fractionated prior to LC-MS/MS analysis (Fig. 1b). To elucidate the regulatory network of ZNF322A in lung cancer cells, we analyzed the proteomic and phosphoproteomic profiles for biological function, pathway, and kinase activity using A549 lung cancer to explore the effect and roles of ZNF322A (Fig. 1). In total, we identified 2754 phosphosites on 1822 unique phosphopeptides and mapped to 976 phosphoproteins, with a phosphopeptide enrichment efficacy of $91.4 \%$ (Fig. 2a). Most peptides were singly or doubly phosphorylated, of which $\sim 64 \%$ of peptides were singly phosphorylated (Fig. 2b). The phosphosites were divided into three categories based on

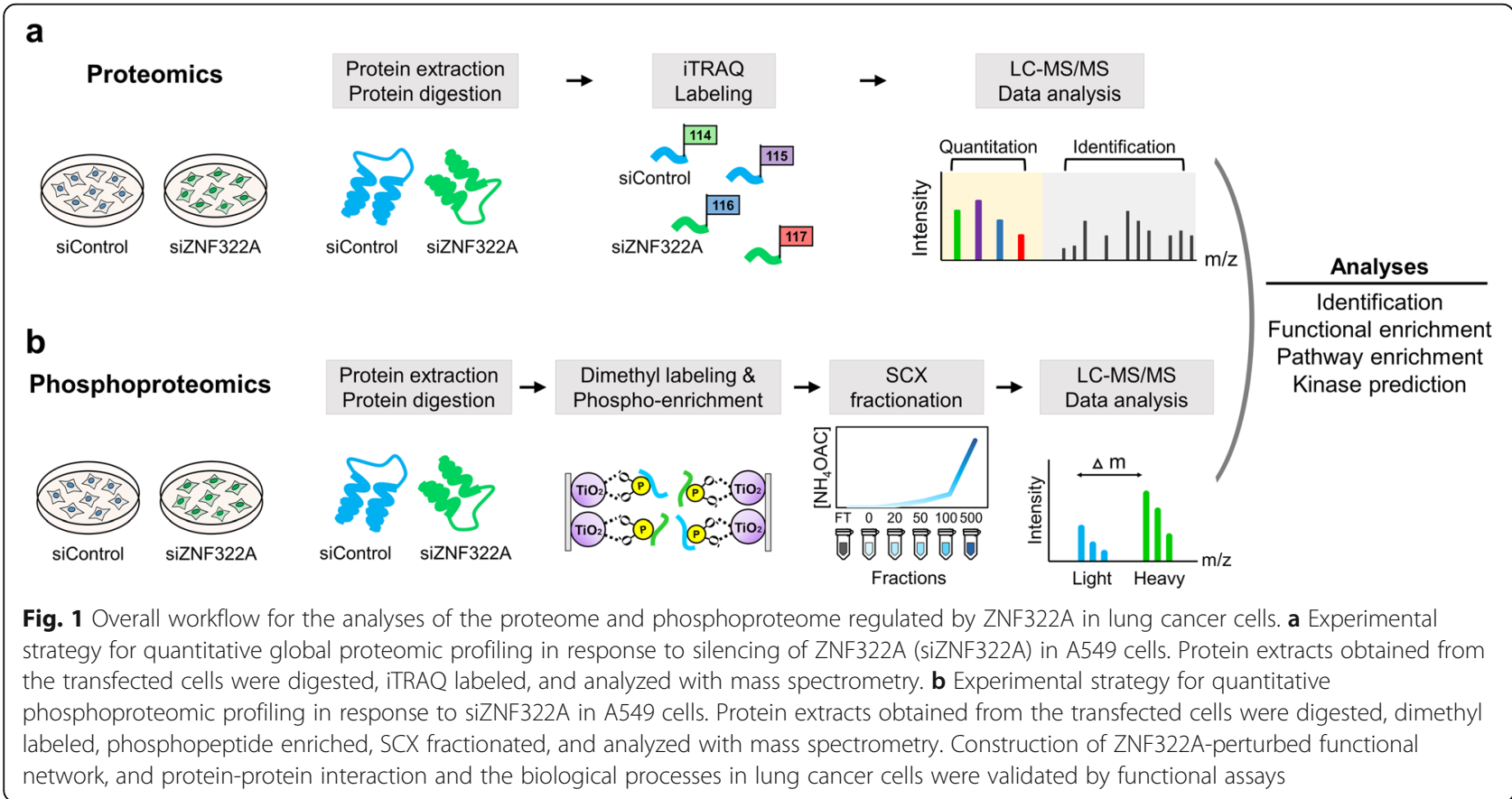


a

Phosphoproteomic profiling

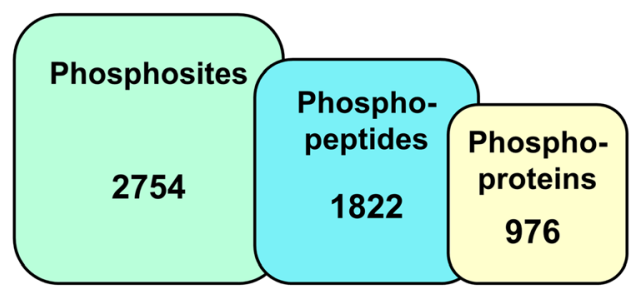

C

Phosphorylation site localization probability

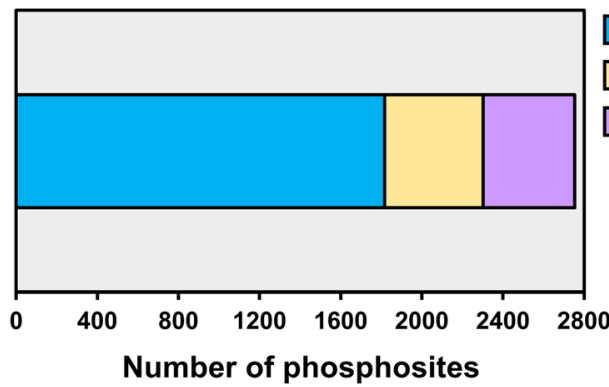

b

Number of singly, doubly, triply \& quadruply phosphorylated peptides

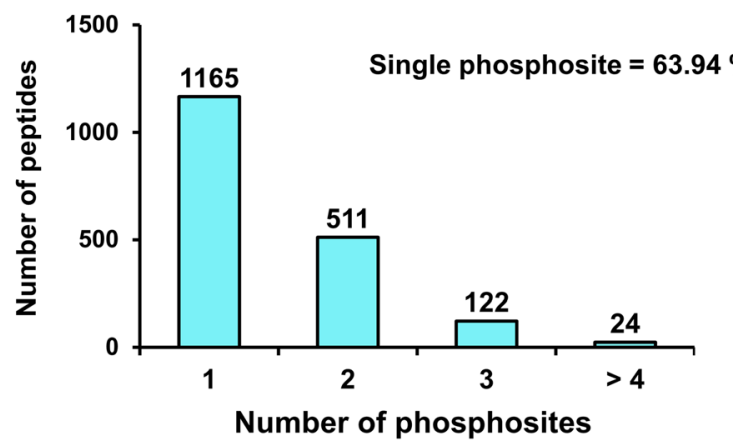

d

\section{Distribution of phosphorylated serine, threonine, \& tyrosine sites}

$P>0.75$

$0.75 \geq P \geq 0.50$

$P<0.50$

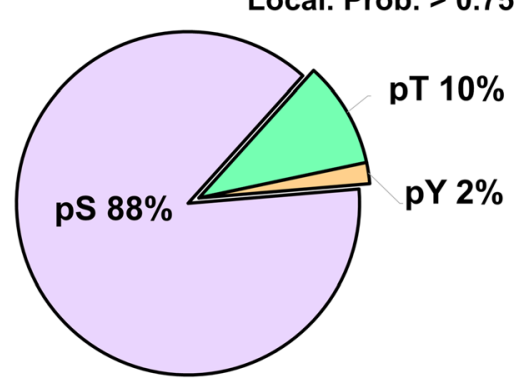

Fig. 2 Phosphoproteomic profile of siZNF322A in A549 lung cancer cells. a Number of identified phosphoproteins, phosphopeptides, and phosphosites of the phosphoproteomics of ZNF322A-silenced A549 cells. b Number of singly, doubly, triply and quadruply phosphorylated peptides. c Localization probability of identified phosphorylated sites. Bar plot showed the percentages of localization probability, of which the majority of localization probability $>0.75$, in ZNF322A-silencing phosphoproteome. $\mathbf{d}$ Distribution of phosphorylated serine, threonine, and tyrosine sites. Pie chart showed the distribution of phosphorylation sites on Ser (88\%) Thr (10\%) and Tyr (2\%) residues

their localization probability $(\mathrm{P})$ : class I $(P>0.75)$, class II $(0.75 \geq P \geq 0.5)$, and class III $(P<0.5)$. Among the 2754 phosphosites, 1817 (65.97\%) phosphosites were assigned with a probability of at least 0.75 (Fig. 2c). Also, the Class I phosphosites revealed a distribution of $88 \%$ phosphoserine (pSer), $10 \%$ phosphothreonine (pThr), and $2 \%$ phosphotyrosine residues (pTyr) (Fig. 2d). To assess an insight of ZNF322A-silencing phosphoproteomic profile, the quantified phosphosites ratio with high localization probability (Class I), were depicted in histogram of $\log _{2}$ transformed ratios (Additional file 1: Fig. S2). The distributions of phosphosite ratios follow normal distribution with the mean equals to -0.02 and the standard deviation equals to 0.41 . Phosphosites that showed 1.5 -fold or greater changes in siZNF322A cells were considered to be up-regulated phosphosites, while those less than 0.67 -fold changes were considered to be down-regulated phosphosites. Our phosphoproteomics quantified 45 upregulated phosphosites and 72 down-regulated phosphosites corresponding to 42 and 61 phosphoproteins, respectively (Additional file 2: Table S2).

Functional networks of ZNF322A-mediated proteins and protein phosphorylation

To establish the functional roles of ZNF322A in lung cancer, we analyzed phosphoproteomic and our previous proteomic studies [7] to perform Gene Ontology (GO) and pathway enrichment. To reveal ZNF322Amediated biological processes in lung cancer, we performed GO enrichment analyses of 77 proteins and 


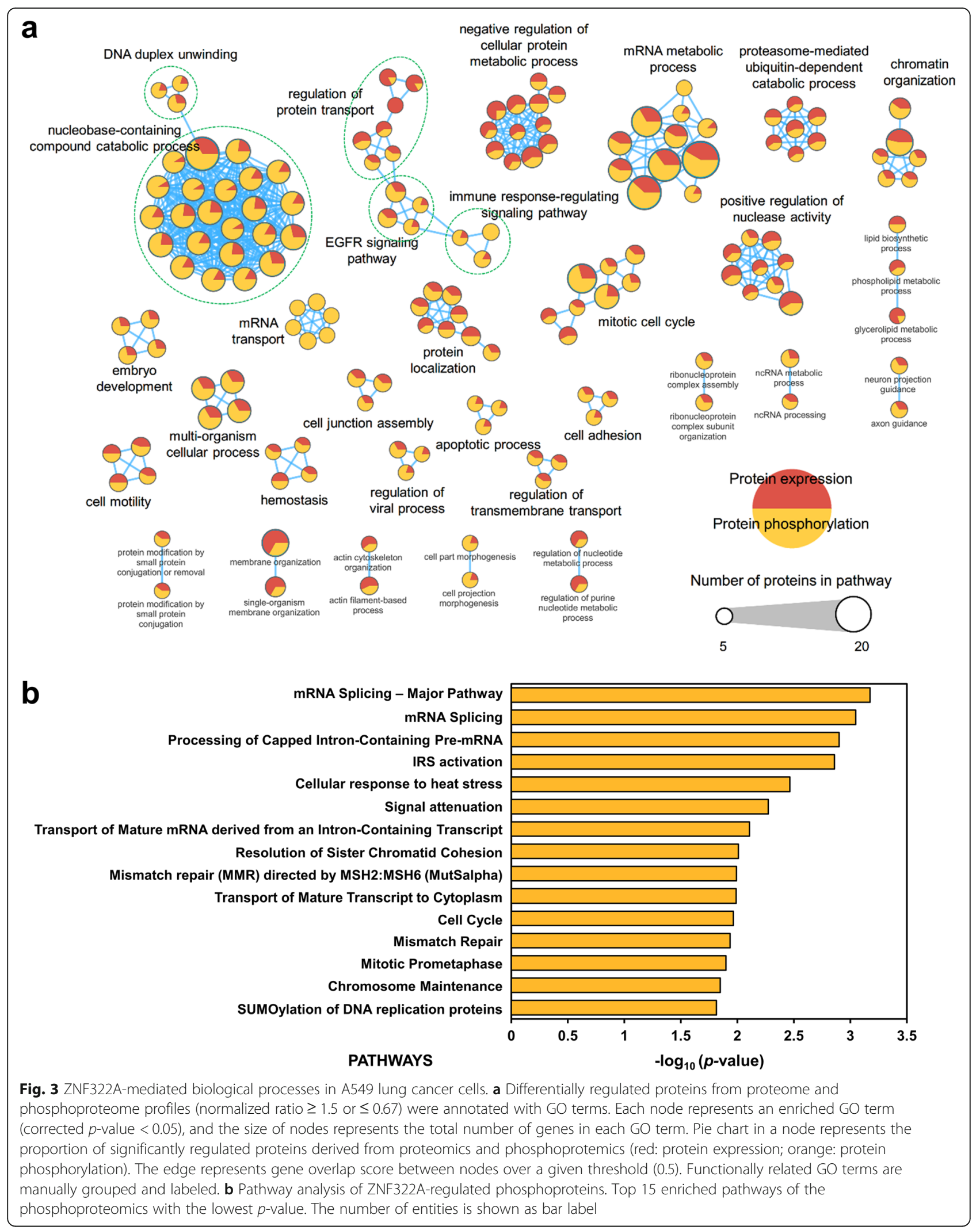


103 phosphoproteins that exhibited significantly changed in proteomics and phosphoproteomics under ZNF322A silencing (Fig. 3a). Consistent to our previous ZNF322A silencing proteomic profile and studies that ZNF322A is related to the regulation of nuclease activity, embryonic development, cell cycle, cell motility, and apoptosis, suggesting ZNF322A-regulated protein phosphorylation and signal transduction also play a pivotal role in lung cancer progression (Fig. 3a, Additional file 2: Table S3). Next, we investigated the signaling pathways specifically triggered or modulated by ZNF322A at the protein phosphorylation level, the differentially phosphorylated proteins were employed in pathway enrichment analysis, apparently the top 15 enriched pathways were mostly related to RNA processing, chromosome organization and maintenance, and cell cycle (Fig. 3b, Additional file 2: Table S4). Interestingly, IRS activation, cellular response to heat stress, and signal attenuation arose our further interest to investigate the association to ZNF322A.

\section{ZNF322A modulates glucose uptake via the IRS1/PI3K/ AKT pathway in lung cancer cells}

To elucidate the ZNF322A-regulated phosphorylated protein network in response to insulin activation (Fig. $3 \mathrm{~b})$, we analyzed our previous anti-ZNF322A ChIPseq data [9], and found the upstream kinase, serine/ threonine-protein kinase pim-3 (PIM3), of Insulin receptor substrate 1 (IRS1) had ZNF322A binding signals [35]. As ZNF322A has been suggested to transcriptionally regulate expression of PIM3, qRTPCR and immunoblotting were performed on A549 lung cancer cells with ZNF322A perturbation (Fig. 4a). Silencing of ZNF322A significantly upregulated the mRNA (Fig. 4b) and protein (Fig. 4c and d) expressions of PIM3. These results suggest the inhibitory transcriptional regulation of ZNF322A on PIM3 in A549 lung cancer cells. Moreover, ZNF322A binding signals were found to be located at IRS1 promotor in anti-ZNF322A ChIP-seq profile [9], suggesting a transcriptionally regulation of ZNF322A on IRS1 (Additional file 1: Fig. S3). IRS1 is one of the key mediator for glucose metabolism that regulates translocation of glucose transporter through the PI3K/AKT signaling pathway to facilitate glucose uptake $[19,36-$ 38]. Phosphorylation of IRS1 at serine ${ }^{1101}$ participates in suppressing insulin activation via inhibition of PI3K/AKT signaling [39]. Based on the above findings, we identified a phosphorylation residue, serine $^{1101}$, in IRS1 with a 1.54-fold changed upon ZNF322A silencing of A549 lung cancer cells in the quantitative phosphoproteomic data (Additional file 2: Table S2). To ascertain the stimulatory effect of ZNF322A on lung cancer in the IRS1/PI3K/AKT pathway, we further analyzed IRS1 and AKT expressions of ZNF322A-silenced A549 cells at $48 \mathrm{~h}$ posttransfection by immunoblotting (Fig. 4e). The quantified results showed that IRS1 phosphorylation at serine ${ }^{1101}$ was significantly upregulated, and the phosphorylation of serine ${ }^{473}$ in AKT was significantly downregulated in ZNF322A-silencing lung cancer cells (Fig. 4f). Since the IRS1/PI3K/AKT signaling pathway participates in the process of glucose transport into cells, we further examined glucose uptake by a fluorescent glucose analog, 2-NBDG, to determine the effect of ZNF322A in lung cancer. 2-NBDG fluorescent levels were monitored by fluorescence microscopy after transfection with siZNF322A for $48 \mathrm{~h}$ (Fig. 4g). The fluorescent intensity and number of 2NBDG uptake were significantly decreased in ZNF322A-silenced cells (Fig. 4h). Taken together, we purpose that the depletion of ZNF322A in A549 lung cancer cells transcriptionally regulates PIM3 kinase to induce IRS1 ${ }^{\text {Ser1101 }}$ phosphorylation, which attenuates PI3K/AKT signaling pathway and inhibits $\mathrm{AKT}^{\mathrm{S} 473}$, leading to glucose uptake blockade (Fig. 4i). This is the first time to discover that silencing of ZNF322A is capable to mediate the regulation of insulin signal transduction pathway and caused glucose starvation in lung cancer cells.

\section{ZNF322A mediates heat shock protein 27 phosphorylation and elicits unfolded protein response} We next sought to determine whether the ZNF322A is linked to cellular response to heat stress in lung cancer (Fig. 3b). Heat stress causes the accumulation of misfolded proteins in the endoplasmic reticulum (ER), which leads to activation of the unfolded protein response (UPR), as phosphorylation of eIF $2 \alpha$ at serine ${ }^{51}$ indicates that UPR is triggered to attenuate protein synthesis [40]. The perturbation of UPR was validated by protein phosphorylation of serine ${ }^{51}$ on eIF $2 \alpha$ which were increased by 1.9-fold changed upon ZNF322A silencing (Fig. 5a and b). To investigate the regulatory network of ZNF322A, we analyzed the significantly regulated proteins, phosphoproteins, and predicted kinases into a protein-phosphoprotein-kinase interaction (PPKI) network (Additional file 1: Fig. S4). The kinase activity analysis provided us a robust path to predict the complicated kinase-substrate relationship, and a total of eighty-four kinases were enriched using DynaPho [31]. HSP27 is a substrate of MAPK-activated protein kinase 2 (MAPKAPK2), participating in p38 MAPK pathway [41]. MAPKAPK2 is the predicted kinase to the sequence window of HSP27 mass spectrum, which recognized a short linear sequence of amino acids with overrepresented motif patterns for initiating phosphorylation at HSP27 serine ${ }^{82}$ (Fig. 5c). Thus, we further 


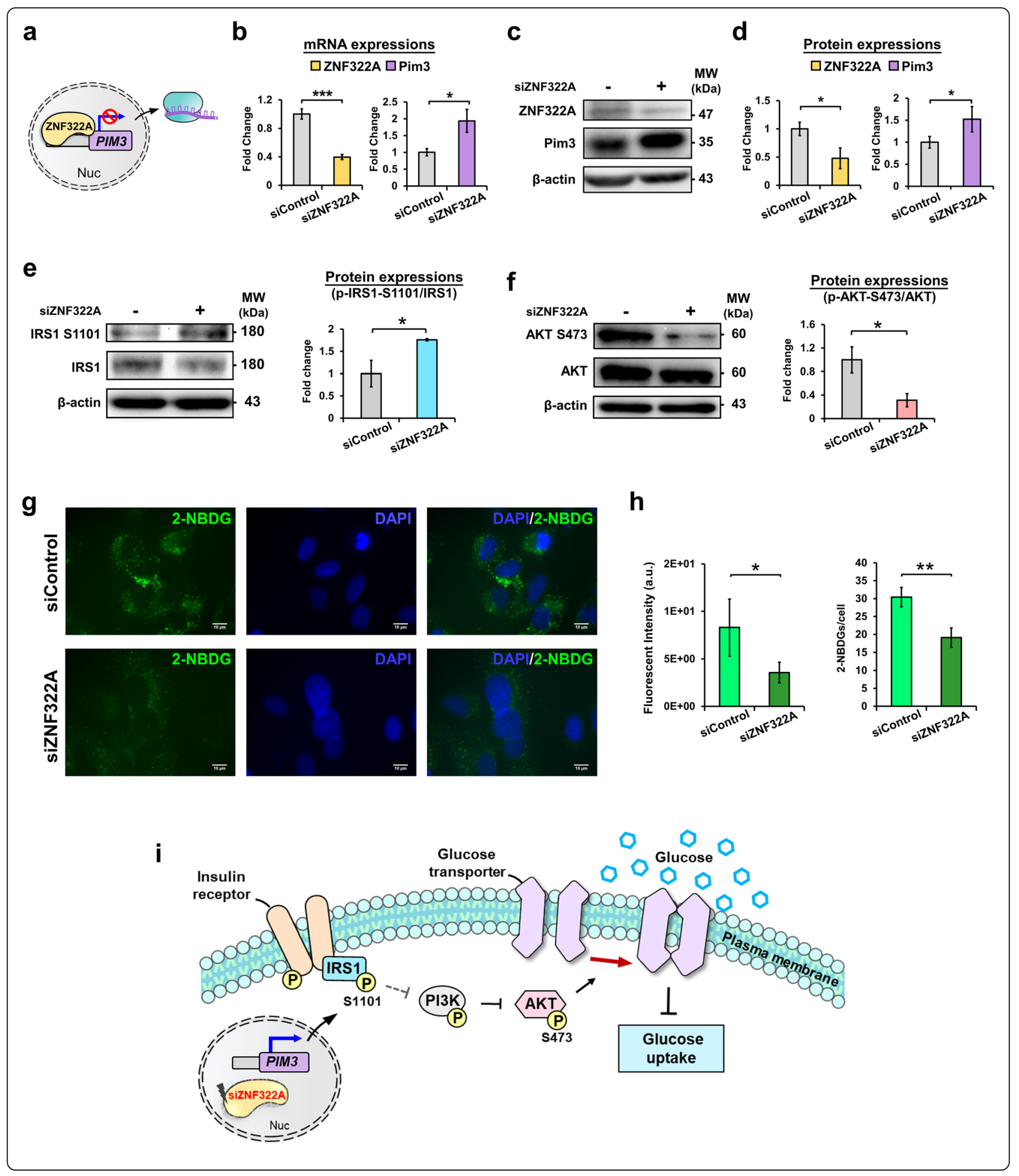


(See figure on previous page.)Fig. 4 ZNF322A modulates glucose uptake via the IRS1/PI3K/AKT pathway in A549 lung cancer cells. a Schematic representation of the inhibitory transcription regulation of ZNF322A on PIM3. $\mathbf{b}$ Relative mRNA levels of ZNF322A and PIM3 were examined by RT-qPCR analysis, and normalized by GAPDH. c-d Proteins were extracted from ZNF322A-silenced (siZNF322A) A549 cells after $48 \mathrm{~h}$ transfection. Protein expressions of ZNF322A and PIM3 were examined by immunoblot analysis. Representative western blot (c) and associated densitometric analysis (d) for ZNF322A and PIM3 expressions in A549 lung cancer cells. e-f Protein expressions of IRS1 ${ }^{\text {S1101 }}$ and AKT ${ }^{\text {S473 }}$ phosphorylation were examined by immunoblot analysis. Phosphorylation levels were determined and normalized to the level of total proteins. The normalized values were compared between siRNA control (siControl) and siZNF322A groups. $\mathbf{g}$-h Effect of 2-NBDG uptake upon ZNF322A perturbation in A549 lung cancer cells. Representative fluorescence microscopy images of 2-NBDG uptake ability in siRNA control (upper) and siZNF322A-silenced (bottom) A549 cells. 2-NBDG: green; DAPI: blue; scale bar: $10 \mu \mathrm{m}$ (g). Mean fluorescence intensity (left) and number (right) of 2-NBDG in siControl and siZNF322A cells (h). (i) Schematic representation of the depletion of ZNF322A by siRNAs in A549 lung cancer cells transcriptionally regulates PIM3

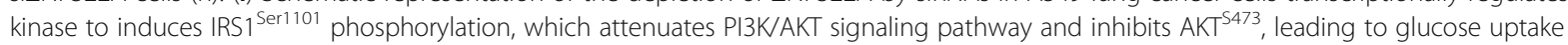
blockade. $\beta$-actin is the internal control to normalize protein expression. The bars represent densitometric analysis of three biological replicates and the data are shown as mean \pm SD. ${ }^{*} p<0.05$; ${ }^{* *} p<0.01 ;{ }^{* * *} p<0.001$

ascertained our mass spectrometric observation with immunoblot analysis using a phospho-specific antibody against serine $^{82}$ on HSP27 (Fig. 5d). The immnuoblot results showed a significantly increased of HSP27 at serine $^{82}$ with a 4.2 -fold changed at $48 \mathrm{~h}$ siZNF322A in lung cancer (Fig. 5e). The intracellular concentration of the HSP27 increases after heat and metabolic stresses [42]. Besides, HSP27 is involved in ER stress and capable to bind to improperly folded proteins and further transfer them into protein degradation machinery such as autophagosomes $[43,44]$. The above evidence and findings suggest that knockdown of ZNF322A may suppress lung cancer proliferation through HSP27 at serine ${ }^{82}$ phosphorylation, and induces UPR (Fig. 5f).

\section{Silencing of ZNF322A induces autophagosome formation in lung cancer cells}

ZNF322A modulated the IRIS/PI3K/AKT and HSP27/ MAPK pathways to perturb glucose uptake and UPR upon ZNF322A-slienced A549 lung cancer cells, and

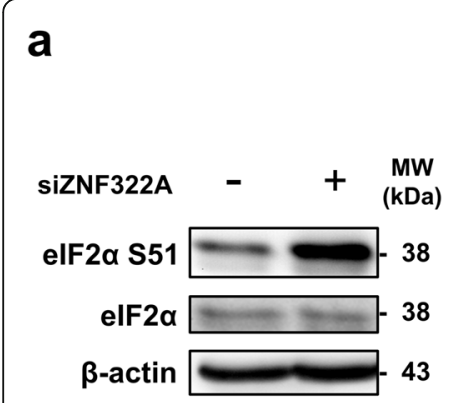

b
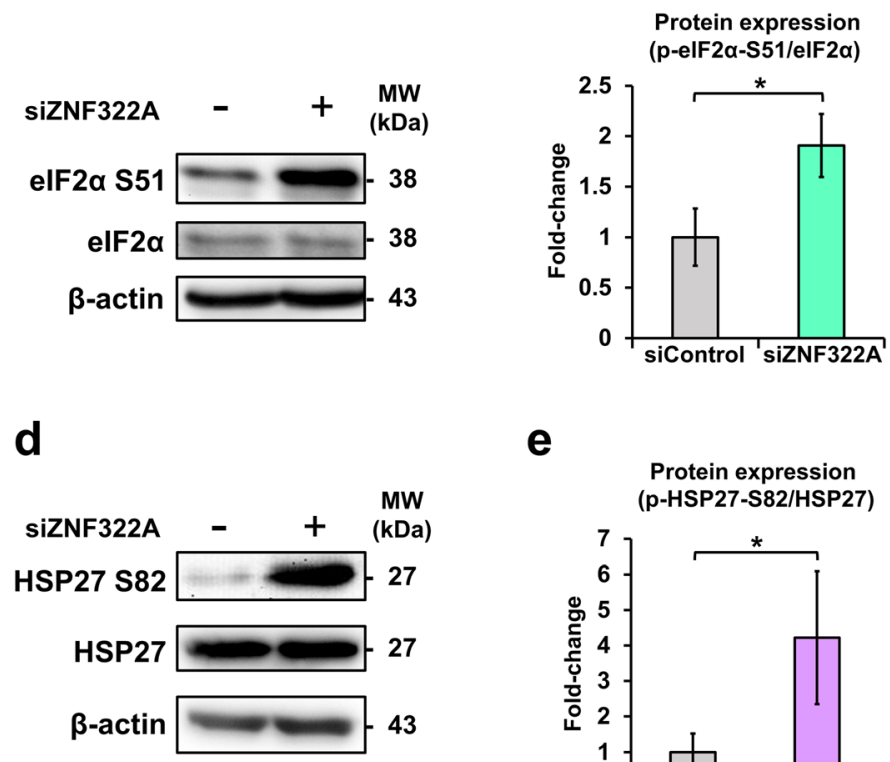

e

Protein expression (p-HSP27-S82/HSP27)
C


f

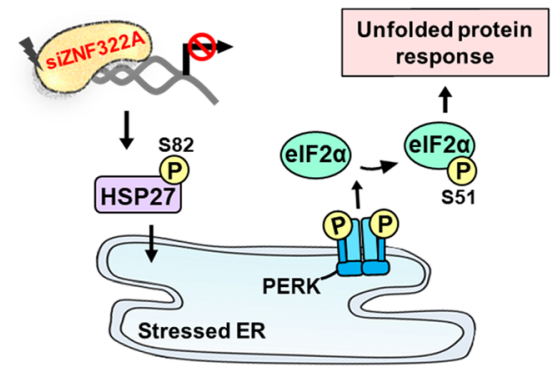

Fig. 5 ZNF322A mediates heat shock protein 27 phosphorylation and elicits unfolded protein response. a The protein expressions of elF2a in UPR signaling pathway were analysis by immnoblotting. b Phosphorylation of elF $2 a^{\mathrm{S} 51}$ was determined and normalized to the level of total elF2a. The normalized values were then compared between siControl and siZNF322A. c Enriched consensus motifs of MAPKAPK2 on HSP27 ${ }^{582}$ were predicted and identified in ZNF322A-silenced phosphoproteome. MS/MS spectrum for HSP27 (QIpSSGVSEIR), and fragment ions in the MS/MS spectrum localize at serine ${ }^{82}$ in HSP27. d Validation of HSPB1 ${ }^{\text {S82 }}$ phosphorylation using immunoblot analysis. e Phosphorylation of HSP27 was determined and normalized to the level of total HSP27. The normalized values were compared between siControl and siZNF322A groups. $\mathbf{f}$ Schematic representation of the heat stress responses of HSP27 ${ }^{582}$ via UPR pathway by silencing of ZNF322A in A549 lung cancer. $\beta$-actin is the internal control to normalize protein expression. The bars represent densitometric analysis of three biological replicates and the data are shown as mean \pm SD. * $p<0.05$ 
the cumulative evidence also suggest that suppression of the PI3K/AKT/mTOR pathway and induction of HSP27-regulated misfolded proteins triggers cytoprotective autophagy in cancer [44-47], hence, we further investigated whether autophagy was mediated by ZNF322A in lung cancer. First, we assessed mTOR phosphorylation to determine mTOR activity upon ZNF322A-silencing A549 cells, and found phosphorylation of serine ${ }^{2448}$ in mTOR and mTOR significantly decreased by siZNF322A (Fig. 6a and b). The results revealed that silencing of ZNF322A represses mTOR activity, thereby promoting autophagy. Next, we determined the expression levels of autophagy-related proteins including microtubule-associated protein 1 light chain-3B (LC3B) and sequestosome 1 (SQSTM1/p62) after 24 and $48 \mathrm{~h}$ under ZNF322A silencing (Fig. 6c), and found that the ratios of LC3B11/LC3B I and SQSTM1 were significantly increased, suggesting the inhibition of ZNF322A may facilitate cellular autophagy in lung cancer (Fig. 6d). Consistently, transmission electron microscopy (TEM) images of A549 lung cancer cells clearly showed the formation of double and multilayered membrane-bounded structures, which signify autophagosomes were formed in the siZNF322A compared to the siControl at 24 and $48 \mathrm{~h}$ transfection (Fig. 6e). Autophagy processes start with the formation of double- and multi-membrane autophagosome, and mature and fuse with lysosome to generate autolysosome. The morphological features of autophagic vacuoles manifested cell death figures and typically increased autophagosomes and autolysosomes numbers, as well as rupture and blebbing of plasma membranes were observed in the siZNF322A cells in our study (Fig. 6f). mTOR is a key component that coordinately regulates the balance between cell growth and autophagy in response to cellular physiological conditions and environmental stress. Upon stress, mTOR is inhibited, leading to the induction of autophagy in cells [48]. Taken together, we observed autophagic vacuolization, which is commonly observed in cells undergoing apoptotic or necrotic cell death, this observation hints that cell death under silencing of ZNF223A associates with autophagy mechanism via IRIS/PI3K/AKT and HSP27/ MAPK pathways.

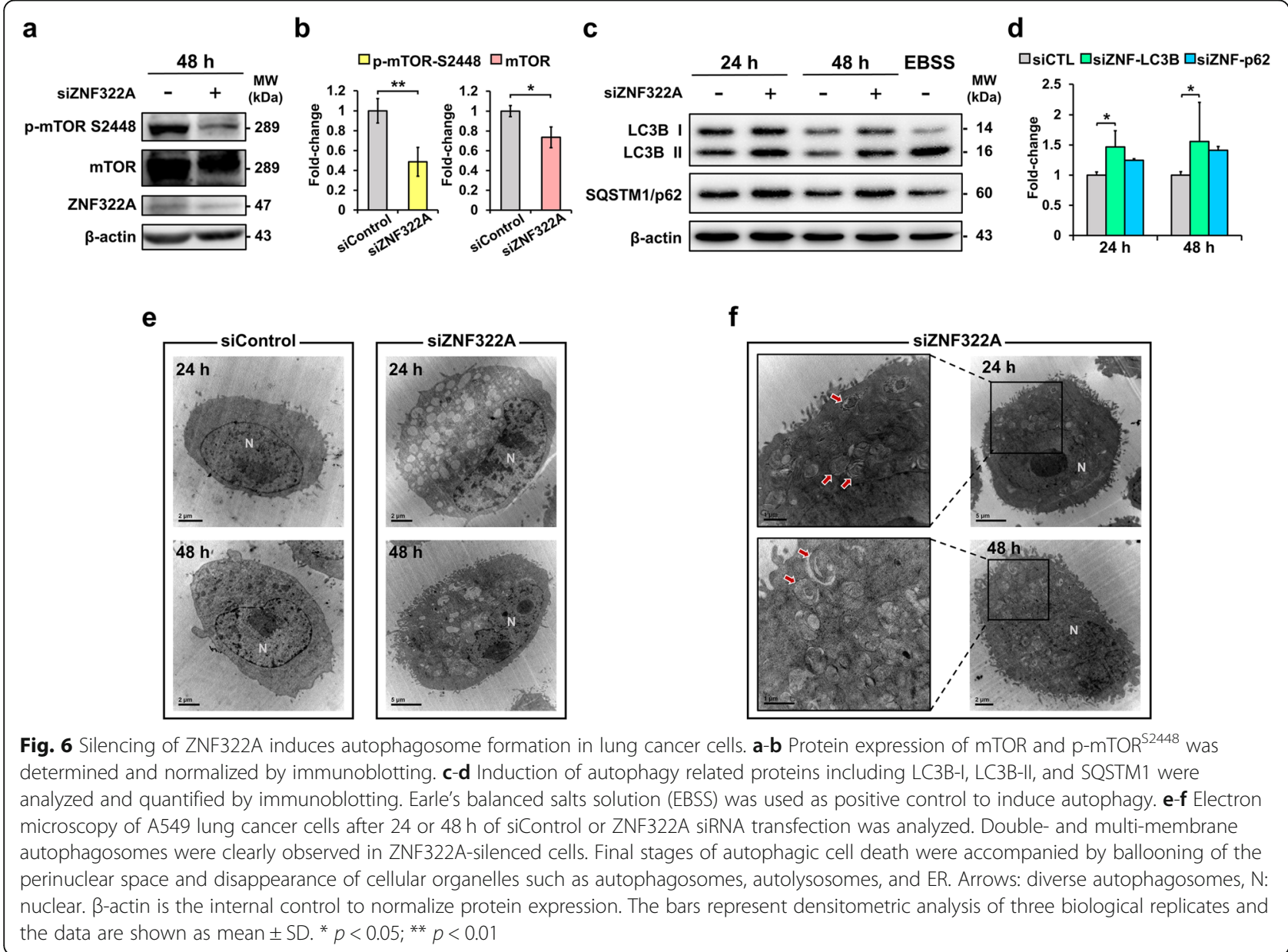




\section{Discussion}

ZNF proteins, as a huge gene family, exert distinct functions in several aspects of cellular processes, including differentiation, proliferation, apoptosis and metastasis [2-4]. Overexpression of ZNF322A promoted tumor growth and metastasis in both cell-based and animalbased studies, moreover, ZNF322A was significantly overexpressed in lung cancer patients and associated with poor overall survival, suggesting ZNF322A is an oncogene in lung cancer $[7,8]$. Our previous studies have been using proteomic approach to investigate the regulated proteins in different network and disease. Consistent with our previous reports, RNA processing and RNA splicing were observed to be involved in the ZNF322A downstream signaling pathway, and the functional enrichment also identified other processes such as regulation of protein transport, hemostasis, and metabolic process by analysis the differentially regulated phosphoproteins and proteins individually.

In the REACTOME pathway enrichment analysis, IRS activation was discovered as an induced pathway upon ZNF322A silencing. Insulin receptor substrate 1 (IRS1) and growth factor receptor-bound protein 10 (GRB10) were identified in our phosphoproteomic results, which are participated in IRS activation. IRS1 is a cytoplasmic signaling adapter protein that is able to integrate different signaling cascades by activation through cell surface receptors, including the insulin, insulin-like growth factor 1 (IGF-1), growth hormone (GH) receptors [49]. Phosphorylation of IRS1 on tyrosine residues is required for insulin-stimulated responses, while phosphorylation of IRS1 on serine residues has a dual role, either to enhance or to terminate the insulin effects $[50,51]$. Several epithelial tumors depend constitutive activation of this pathway to increase their glucose supply [32]. IRS1 ${ }^{\mathrm{S} 1101}$ is phosphorylated by protein kinase $\mathrm{C}$ theta $(\mathrm{PKC} \theta)$, Pim and ribosomal protein S6 kinase beta-1 (RPS6KB1) [32, 35, 39, 52]. PKC $\theta$ phosphorylates serine residues on IRS1, which dissociates from the insulin receptor, leading to decreased signaling via PI3K/AKT and reduced glucose uptake. On the other hand, GRB10 also disrupts the association of IRS1 with insulin receptor and further inhibit PI3K/AKT pathway [53]. GRB10 ${ }^{\text {S104 }}$ was also identified upregulated in ZNF322A-silenced cells, but the phosphosite has not yet been annotated. In our study, we suggested that knock-down of ZNF322A in lung cancer induces phosphorylation of IR1S that attenuates $\mathrm{PI} 3 \mathrm{~K} / \mathrm{AKT} / \mathrm{mTOR}$ signaling pathway, leading to glucose starvation which may trigger autophagosome formation. Moreover, we also assessed the phosphorylation level of IRS1 at serine ${ }^{1101}$ in H1299 cell line to establish the effect of ZNF322A on NSCLC. Phosphorylation of IRS1 ${ }^{\mathrm{S} 1101}$ increased in ZNF322Asilenced H1299 lung cancer cells which suggests
ZNF322A is a regulator for IRS1/AKT/mTOR signaling pathway in NSCLC (Additional file 1: Fig. S5).

On the other hand, HSP27 is a $27 \mathrm{kDa}$ molecular chaperone belongs to small heat shock family which is responsible for ER stress and cellular response to heat stress [42, 54-56]. Phosphorylation of HSP27 was found to be contributed to inhibit cancer cell growth due to its conformational changes, leading complex dissociation, and subsequent loss of chaperone activity [57-59]. Consistent with our findings, phosphorylation of HSP27 at serine $^{82}$ was identified in siZNF322A A549 cells from our quantitative phosphoproteomics. In addition, we also validated the phosphorylation of HSP27 at serine ${ }^{82}$ in H1299 cell line to establish the effect of ZNF322A on NSCLC. Phosphorylation of $\mathrm{HSP} 27^{\mathrm{S} 82}$ increased in ZNF322A-silenced H1299 lung cancer cells which indicates that ZNF322A may trigger the HSP27/MAPK signaling pathway in NSCLC (Additional file 1: Fig. S5). In our phosphoproteomic profile, phosphorylation of heat shock protein HSP 90-alpha (HSP90AA1) at serine $^{231}$ was also identified with 11.06 fold-changed upon silencing of ZNF322A, which may contribute to autophagy, however the phosphosite-specific antibody has not yet been established. As previously reported, ZNF322A may act as regulator in gene transcription mediated by the MAPK signaling pathways [60]. Consistent with our findings that siZNF322A upregulated the phosphorylation of HSP27 and MAPKAPK2, participating in 38 MAPK pathway which is responsible for the autophagosome formation.

Collectively, we proposed glucose starvation and UPR in siZNF322A lung cancer cells altered mTOR signaling pathway and further induced autophagosome formation. In the current studies, mTOR has emerged as a critical effector commonly deregulated in human cancer, especially in NSCLC [61]. mTOR pathway is a member of the PI3K cell survival pathway and plays an important role in the regulation of cell growth and proliferation [62]. Another crucial role of mTOR pathway is the AKT, which is activated downstream of growth factor-stimulated PI3K activity and phosphorylates a variety of upstream and downstream substrates. Recently, we demonstrated a novel oncogenic regulatory mechanism of phosphorylation by AKT in regulating ZNF322A protein stability and transcriptional activity that promote lung cancer progression [63]. Autophagy serves as a mechanism of tumor suppression, and as an adaptive stress response in tumor cells to maintain their survival [24, 64], which is a self-destructing homeostatic process that removes damaged organelles, restrained cell growth and non-apoptotic cell death [21]. Studies in the past decades have shown that autophagy can also induce cell death, which occurs in mammalian cells and tissues in response to pathophysiological stimuli [65]. 


\section{Conclusion}

ZNF322A is determined to be an oncogene in lung cancer [66], however, the modulatory role of ZNF322A remained to be clarified. Taking advantage of highthroughput HAMMOC phosphopeptide enrichment and mass spectrometry technology, we are able to profile proteomic and phosphoproteomic data in order to elucidate the ZNF322A-regulated downstream phosphoproteins using integrative analyses. In this study, the two proteomic profiles were generated in different timeframe, which may cause a low overlapping of two proteome datasets. Thus, the differentially regulated proteins of both datasets were used for the analysis of pathway enrichment and PPKI network to establish the pivotal roles of ZNF322A in the protein and protein phosphorylation levels. Variety of biological functions and ZNF322A-mediated kinase-substrate phosphorylation network were revealed in this study. Remarkably, $\mathrm{IRS1}^{\mathrm{S} 1101}$ and $\mathrm{HSP} 27^{\mathrm{S} 82}$ were significantly increased, which was considered as the key downstream targets of ZNF322A. Using bioinformatics approaches including functional enrichment, network analysis and phosphorylation motif analysis, we further showed the involvement of mTOR signaling pathway, and demonstrated that ZNF322A silencing in lung cancer cells induced autophagic cell death, but more underlying mechanism requires detailed investigation. The overall schematic mechanism is illustrated in Fig. 7 and indicates that ZNF322Asilenced A549 lung cancer cells trigger autophagy by glucose starvation and UPR through phosphorylation of $\mathrm{IRS1}^{\mathrm{S} 1101}$ and $\mathrm{HSP} 27^{\mathrm{S} 82}$ to inhibit PI3K/AKT/mTOR pathways and to elicit heat stress. Our results not only provide useful information for phosphoproteomic research in ZNF322A but also reveal a new picture of

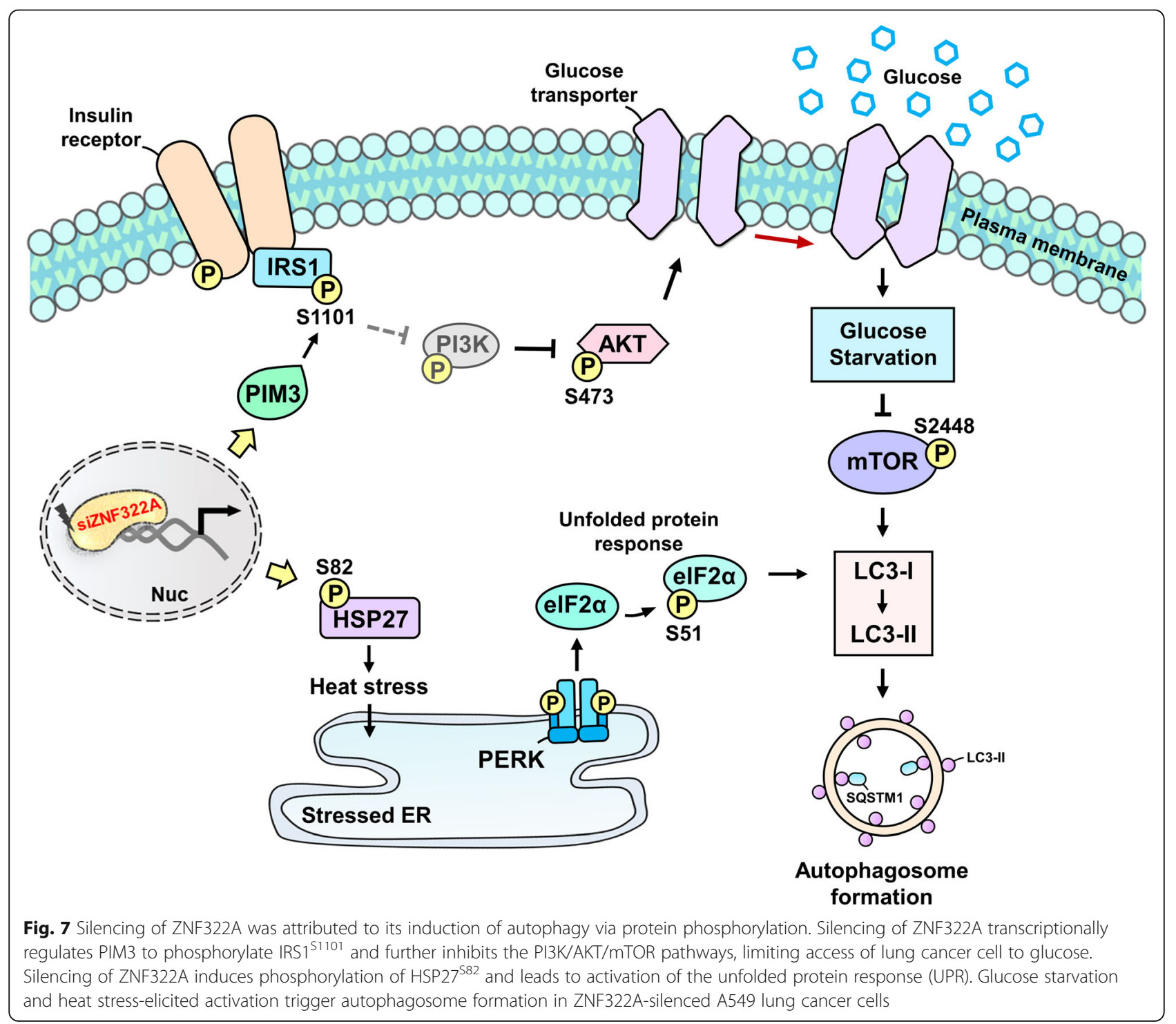


signal transduction corresponding to ZNF322A regulation, and provides insights for further investigation of protein phosphorylation status in ZNF322A-mediated lung cancer. Overall, our data suggest that therapeutic strategies such as efficient delivery of ZNF322A interference RNAs or treatment of targeted inhibitors of ZNF322A downstream post-transcriptional modification can be a potential molecular therapeutic method for lung cancer in the future.

\section{Supplementary information}

Supplementary information accompanies this paper at https://doi.org/10. 1186/s12929-020-00668-5.

Additional file 1: Figure S1. mRNA and protein expressions of
ZNF322A in ZNF322A silenced A549 lung cancer cells. Figure S2. Distribution of phosphorylated site ratios. Figure $\mathbf{S 3}$. Snapshot of the ChIP-seq binding profile of ZNF322A at IRS1 gene in lung cancer cell. Figure S4. Analysis of protein-phosphoprotein-kinase interactions in ZNF322A silenced A549 lung cancer cells. Figure S5. IRS1 $1^{\mathrm{S1101}}$ and $\mathrm{HSP} 27^{\text {S82 }}$ phosphorylation in ZNF322A silenced H1299 lung cancer.

Additional file 2: Table S1. Sequence of primers and annealing conditions for real-time quantitative PCR. Table S2. The list of significantly regulated phosphosites of siZNF322A in A549 lung cancer cells. Table S3. Gene list of Gene Ontology analysis of siZNF322A in A549 lung cancer cells. Table S4. Signaling pathway enrichment analysis of siZNF322A phosphoproteomics in A549 lung cancer cells by REACTOME.

\begin{abstract}
Abbreviations
2-NBDG: 2-(N-(7-nitrobenz-2-oxa-1,3-diazol-4-yl)amino)-2-deoxyglucose; Add1: Alpha-adducin; AKT: Protein kinase B; $\mathrm{C} 2 \mathrm{H} 2$ : Cysteine-2/histidine-2; CCND1: Cyclin D1; CTND1: Catenin delta-1; HAMMOC: Hydroxy acid-modified metal oxide chromatography; HSP27: Heat shock protein beta-1; IRS1: Insulin receptor substrate 1; iTRAQ: Isobaric tag for relative and absolute quantitation; LC-MS/MS: Liquid chromatography-tandem mass spectrometry; MAPKs: Mitogen-activated protein kinases; MAPKAPK2: Mitogen-activated protein kinase-activated protein kinase 2; mTOR: Mammalian target of rapamycin; EGFR: Epidermal growth factor receptor; elF2a: Eukaryotic translation initiator factor 2a; ER: Endoplasmic reticulum; ERK: Extracellular signal-regulated kinase; NSCLC: Non-small cell lung cancer; GO: Gene Ontology; JIP4: c-Jun-amino-terminal kinase-interacting protein 4; JNK: c-jun NH2-terminal kinase; nanoLC-MS/MS: Nanoscale liquid chromatographytandem MS; PI3K: Phosphoinositide 3-kinase; PIM3: Serine/threonine-protein kinase pim-3; PPI: Protein-protein interaction; pSer/pS: Phosphoserine; pThr/ pT: Phosphothreonine; pTyr/pY: Phosphotyrosine; PWM: Position weight matrix; PTM: Post-transcriptional modification; qRT-PCR: Quantitative real time polymerase chain reaction; RPS6KB1: Ribosomal protein S6 kinase beta-1; SCX: Strong cation exchange; siRNA: Small interfering RNA;

TEM: Transmission electron microscopy; UPR: Unfolded protein response; ZNF: Zinc finger
\end{abstract}

\section{Acknowledgments}

We thank Technology Commons in College of Life Science, National Taiwan University and the Core Facilities of Translational Medicine of the National Biotechnology Research Park, Taiwan, for providing technical services.

\section{Authors' contributions}

C.H.Y.C., T.-Y. L. and W.-T.C. performed the experiments. C.-L.H. completed the functional enrichment and network analyses. C.H.Y.C., C.-L.H., T.-Y. L., W.-T.C., Y.-C. W, H.-C. H. and H.-F. J. designed experiments, interpreted the results and wrote the manuscript. H.-C. H. and H.-F. J. conceived the study. The author(s) read and approved the final manuscript.

\section{Funding}

This work was supported by the Ministry of Science and Technology in Taiwan (MOST 104-2627-B-002-001, MOST 106-2320-B-002-053-MY3, MOST107-2221-E-010-017-MY2, and MOST 108-2321-B-006-002).

\section{Availability of data and materials}

The raw data supporting this article is available in the ProteomeXchange repository, Project accession: PXD015936; http://www.ebi.ac.uk/pride.

\section{Ethics approval and consent to participate}

Not applicable.

\section{Consent for publication}

Not applicable.

\section{Competing interests}

The authors declare no competing financial interests.

\section{Author details}

${ }^{1}$ Department of Life Science, National Taiwan University, Taipei 10617, Taiwan. ${ }^{2}$ Department of Medical Research, National Taiwan University Hospital, Taipei 10002, Taiwan. ${ }^{3}$ Department of Pharmacology, College of Medicine, National Cheng Kung University, Tainan 70101, Taiwan. ${ }^{4}$ Institute of Basic Medical Sciences, College of Medicine, National Cheng Kung University, Tainan 70101, Taiwan. ${ }^{5}$ Institute of Biomedical Informatics, National Yang-Ming University, No.155, Sec.2, Linong Street, Taipei 11221, Taiwan. ${ }^{6}$ Department of Life Science, Graduate Institute of Biomedical Electronics and Bioinformatics, National Taiwan University, No. 1, Sec. 4, Roosevelt Road, Taipei 10617, Taiwan.

Received: 6 January 2020 Accepted: 9 June 2020

Published online: 23 June 2020

\section{References}

1. Wolfe SA, Nekludova L, Pabo CO. DNA recognition by Cys2His2 zinc finger proteins. Annu Rev Biophys Biomol Struct. 2000;29:183-212.

2. Tian C, Xing G, Xie P, Lu K, Nie J, Wang J, et al. KRAB-type zinc-finger protein Apak specifically regulates p53-dependent apoptosis. Nat Cell Biol. 2009:11(5):580-91.

3. Jheon AH, Ganss B, Cheifetz S, Sodek J. Characterization of a novel KRAB/ $\mathrm{C} 2 \mathrm{H} 2$ zinc finger transcription factor involved in bone development. J Biol Chem. 2001:276(21):18282-9.

4. Wagner S, Hess MA, Ormonde-Hanson P, Malandro J, Hu H, Chen M, et al. A broad role for the zinc finger protein ZNF202 in human lipid metabolism. J Biol Chem. 2000;275(21):15685-90.

5. Chauhan S, Goodwin JG, Chauhan S, Manyam G, Wang J, Kamat AM, et al. ZKSCAN3 is a master transcriptional repressor of autophagy. Mol Cell. 2013; 50(1):16-28.

6. Meruvu S, Hugendubler L, Mueller E. Regulation of adipocyte differentiation by the zinc finger protein ZNF638. J Biol Chem. 2011;286(30):26516-23.

7. Jen J, Lin LL, Chen HT, Liao SY, Lo FY, Tang YA, et al. Oncoprotein ZNF322A transcriptionally deregulates alpha-adducin, cyclin D1 and p53 to promote tumor growth and metastasis in lung cancer. Oncogene. 2016;35(18):2357-69.

8. Liao SY, Chiang CW, Hsu CH, Chen YT, Jen J, Juan HF, et al. CK1delta/ GSK3beta/FBXW7alpha axis promotes degradation of the ZNF322A oncoprotein to suppress lung cancer progression. Oncogene. 2017;36(41): 5722-33.

9. Jen J, Liu CY, Chen YT, Wu LT, Shieh YC, Lai WW, et al. Oncogenic zinc finger protein ZNF322A promotes stem cell-like properties in lung cancer through transcriptional suppression of c-Myc expression. Cell Death Differ. 2019;26(7):1283-98.

10. Lo FY, Chang JW, Chang IS, Chen YJ, Hsu HS, Huang SF, et al. The database of chromosome imbalance regions and genes resided in lung cancer from Asian and Caucasian identified by array-comparative genomic hybridization. BMC Cancer. 2012;12:235.

11. Krystal GW, Honsawek S, Litz J, Buchdunger E. The selective tyrosine kinase inhibitor STI571 inhibits small cell lung cancer growth. Clin Cancer Res. 2000;6(8):3319-26.

12. Reimand J, Wagih $\mathrm{O}$, Bader GD. The mutational landscape of phosphorylation signaling in cancer. Sci Rep. 2013;3:2651. 
13. Dhillon AS, Hagan S, Rath O, Kolch W. MAP kinase signalling pathways in cancer. Oncogene. 2007;26(22):3279-90.

14. Itoh N, Semba S, Ito M, Takeda H, Kawata S, Yamakawa M. Phosphorylation of Akt/PKB is required for suppression of cancer cell apoptosis and tumor progression in human colorectal carcinoma. Cancer. 2002:94(12):3127-34.

15. Macek B, Mann M, Olsen JV. Global and site-specific quantitative phosphoproteomics: principles and applications. Annu Rev Pharmacol Toxicol. 2009;49:199-221.

16. Lim YP. Mining the tumor phosphoproteome for cancer markers. Clin Cancer Res. 2005;11(9):3163-9.

17. Harsha HC, Pandey A. Phosphoproteomics in cancer. Mol Oncol. 2010;4(6): 482-95.

18. Laplante M, Sabatini DM. mTOR signaling in growth control and disease. Cell. 2012:149(2):274-93.

19. Huang X, Liu G, Guo J, Su Z. The PI3K/AKT pathway in obesity and type 2 diabetes. Int J Biol Sci. 2018;14(11):1483-96.

20. Geronimo-Olvera C, Montiel T, Rincon-Heredia R, Castro-Obregon S, Massieu L. Autophagy fails to prevent glucose deprivation/glucose reintroductioninduced neuronal death due to calpain-mediated lysosomal dysfunction in cortical neurons. Cell Death Dis. 2017:8(6):e2911.

21. Kanzawa T, Zhang L, Xiao L, Germano IM, Kondo Y, Kondo S. Arsenic trioxide induces autophagic cell death in malignant glioma cells by upregulation of mitochondrial cell death protein BNIP3. Oncogene. 2005; 24(6):980-91.

22. Shao Y, Gao Z, Marks PA, Jiang X. Apoptotic and autophagic cell death induced by histone deacetylase inhibitors. Proc Natl Acad Sci. 2004;101(52): 18030-180305.

23. Levy JM, Thorburn A. Targeting autophagy during cancer therapy to improve clinical outcomes. Pharmacol Ther. 2011;131(1):130-41.

24. Baehrecke EH. Autophagy: dual roles in life and death? Nat Rev Mol Cell Biol. 2005:6(6):505-10.

25. Fumarola C, Bonelli MA, Petronini PG, Alfieri RR. Targeting PI3K/AKT/ mTOR pathway in non small cell lung cancer. Biochem Pharmacol. 2014;90(3):197-207.

26. Chen $M$, Du $Y$, Qui $M$, Wang $M$, Chen $K$, Huang $Z$, et al. Ophiopogonin Binduced autophagy in non-small cell lung cancer cells via inhibition of the PI3K/Akt signaling pathway. Oncol Rep. 2013:29(2):430-6.

27. Rappsilber J, Mann M, Ishihama Y. Protocol for micro-purification, enrichment, pre-fractionation and storage of peptides for proteomics using StageTips. Nat Protoc. 2007;2(8):1896-906.

28. Cheung $\mathrm{CHY}$, Hsu CL, Chen KP, Chong ST, Wu CH, Huang HC, et al. MCM2 regulated functional networks in lung cancer by multi-dimensional proteomic approach. Sci Rep. 2017;7(1):13302.

29. Liu S, Yu F, Yang Z, Wang T, Xiong H, Chang C, et al. Establishment of dimethyl labeling-based quantitative Acetylproteomics in Arabidopsis. Mol Cell Proteomics. 2018:17(5):1010-27.

30. Merico D, Isserlin R, Stueker O, Emili A, Bader GD. Enrichment map: a network-based method for gene-set enrichment visualization and interpretation. PLoS One. 2010;5(11):e13984.

31. Hsu CL, Wang JK, Lu PC, Huang HC, Juan HF. DynaPho: a web platform for inferring the dynamics of time-series phosphoproteomics. Bioinformatics. 2017.

32. Sourbier C, Scroggins BT, Ratnayake R, Prince TL, Lee S, Lee MJ, et al. Englerin a stimulates PKCtheta to inhibit insulin signaling and to simultaneously activate HSF1: pharmacologically induced synthetic lethality. Cancer Cell. 2013;23(2):228-37.

33. Hamilton N. Quantification and its applications in fluorescent microscopy imaging. Traffic. 2009;10(8):951-61.

34. Dell RB, Holleran S, Ramakrishnan R. Sample size determination. ILAR J. 2002; 43(4):207-13

35. Song JH, Padi SK, Luevano LA, Minden MD, DeAngelo DJ, Hardiman G, et al. Insulin receptor substrate 1 is a substrate of the Pim protein kinases. Oncotarget. 2016;7(15):20152-65.

36. Shaw LM. The insulin receptor substrate (IRS) proteins: at the intersection of metabolism and cancer. Cell Cycle. 2011;10(11):1750-6.

37. Pollak M. Insulin and insulin-like growth factor signalling in neoplasia. Nat Rev Cancer. 2008;8(12):915-28.

38. Whelan SA, Dias WB, Thiruneelakantapillai L, Lane MD, Hart GW. Regulation of insulin receptor substrate 1 (IRS-1)/AKT kinase-mediated insulin signaling by O-linked beta-N-acetylglucosamine in 3T3-L1 adipocytes. J Biol Chem. 2010;285(8):5204-11.
39. Tremblay F, Brule S, Hee Um S, Li Y, Masuda K, Roden M, et al. Identification of IRS-1 Ser-1101 as a target of S6K1 in nutrient- and obesity-induced insulin resistance. Proc Natl Acad Sci U S A. 2007;104(35):14056-61.

40. Homma T, Fujii J. Heat stress promotes the down-regulation of IRE1alpha in cells: an atypical modulation of the UPR pathway. Exp Cell Res. 2016;349(1): 128-38.

41. Shiryaev A, Dumitriu G, Moens U. Distinct roles of MK2 and MK5 in CAMP/ PKA- and stress/p38MAPK-induced heat shock protein 27 phosphorylation. J Mol Signal. 2011;6(1):4

42. Landry J, Lambert H, Zhou M, Lavoie JN, Hickey E, Weber LA, et al. Human HSP27 is phosphorylated at serines 78 and 82 by heat shock and mitogenactivated kinases that recognize the same amino acid motif as 56 kinase II. J Biol Chem. 1992:267(2):794-803.

43. Kumano M, Furukawa J, Shiota M, Zardan A, Zhang F, Beraldi E, et al. Cotargeting stress-activated Hsp27 and autophagy as a combinatorial strategy to amplify endoplasmic reticular stress in prostate cancer. Mol Cancer Ther. 2012;11(8):1661-71.

44. Okuno M, Adachi S, Kozawa O, Shimizu M and Yasuda I. The Clinical Significance of Phosphorylated Heat Shock Protein 27 (HSPB1) in Pancreatic Cancer. Int J Mol Sci. 2016;17(1).

45. Janku F, McConkey DJ, Hong DS, Kurzrock R. Autophagy as a target for anticancer therapy. Nat Rev Clin Oncol. 2011;8(9):528-39.

46. Li P, Shi J, He Q, Hu Q, Wang YY, Zhang LJ, et al. Streptococcus pneumoniae induces autophagy through the inhibition of the PI3K-I/Akt/ mTOR pathway and ROS hypergeneration in A549 cells. PLoS One. 2015; 10(3):e0122753.

47. Hung JY, Hsu YL, Li CT, Ko YC, Ni WC, Huang MS, et al. 6-Shogaol, an active constituent of dietary ginger, induces autophagy by inhibiting the AKT/ mTOR pathway in human non-small cell lung cancer A549 cells. J Agric Food Chem. 2009:57(20):9809-16.

48. Schmeisser K, Parker JA. Pleiotropic effects of mTOR and autophagy during development and aging. Front Cell Dev Biol. 2019;7:192.

49. Mardilovich K, Pankratz SL, Shaw LM. Expression and function of the insulin receptor substrate proteins in cancer. Cell Commun Signal. 2009;7:14.

50. Gual P, Le Marchand-Brustel Y, Tanti JF. Positive and negative regulation of insulin signaling through IRS-1 phosphorylation. Biochimie. 2005;87(1):99-109.

51. Vander Heiden MG, Cantley LC, Thompson CB. Understanding the Warburg effect: the metabolic requirements of cell proliferation. Science. 2009; 324(5930):1029-33.

52. Li Y, Soos TJ, Li X, Wu J, Degennaro M, Sun X, et al. Protein kinase C Theta inhibits insulin signaling by phosphorylating IRS1 at Ser (1101). J Biol Chem. 2004;279(44):45304-7.

53. Wick KR, Werner ED, Langlais P, Ramos FJ, Dong LQ, Shoelson SE, et al. Grb10 inhibits insulin-stimulated insulin receptor substrate (IRS)phosphatidylinositol 3-kinase/Akt signaling pathway by disrupting the association of IRS-1/IRS-2 with the insulin receptor. J Biol Chem. 2003: 278(10):8460-7

54. Choi SK, Kam H, Kim KY, Park SI and Lee YS. Targeting Heat Shock Protein 27 in Cancer: A Druggable Target for Cancer Treatment? Cancers (Basel) 2019;11(8).

55. Hansen RK, Parra I, Lemieux P, Oesterreich S, Hilsenbeck SG, Fuqua SA. Hsp27 overexpression inhibits doxorubicin-induced apoptosis in human breast cancer cells. Breast Cancer Res Treat. 1999;56(2):187-96.

56. Stope MB, Weiss M, Preuss M, Streitborger A, Ritter CA, Zimmermann U, et al. Immediate and transient phosphorylation of the heat shock protein 27 initiates chemoresistance in prostate cancer cells. Oncol Rep. 2014;32(6):2380-6.

57. Yasuda E, Kumada T, Takai S, Ishisaki A, Noda T, Matsushima-Nishiwaki R, et al. Attenuated phosphorylation of heat shock protein 27 correlates with tumor progression in patients with hepatocellular carcinoma. Biochem Biophys Res Commun. 2005;337(1):337-42

58. Matsushima-Nishiwaki R, Takai S, Adachi S, Minamitani C, Yasuda E, Noda T, et al. Phosphorylated heat shock protein 27 represses growth of hepatocellular carcinoma via inhibition of extracellular signal-regulated kinase. J Biol Chem. 2008;283(27):18852-60.

59. Li Y, Wang Y, Zhang C, Yuan W, Wang J, Zhu C, et al. ZNF322, a novel human $\mathrm{C} 2 \mathrm{H} 2$ Kruppel-like zinc-finger protein, regulates transcriptional activation in MAPK signaling pathways. Biochem Biophys Res Commun. 2004;325(4):1383-92

60. Ayuk SM and Abrahamse H. mTOR Signaling Pathway in Cancer Targets Photodynamic Therapy In Vitro. Cells. 2019;8(5). 
61. Hassan B, Akcakanat A, Holder AM, Meric-Bernstam F. Targeting the PI3-kinase/ Akt/mTOR signaling pathway. Surg Oncol Clin N Am. 2013;22(4):641-64.

62. Liao SY, Kuo IY, Chen YT, Liao PC, Liu YF, Wu HY, et al. AKT-mediated phosphorylation enhances protein stability and transcription activity of ZNF322A to promote lung cancer progression. Oncogene. 2019;38(41):6723-36.

63. White E, DiPaola RS. The double-edged sword of autophagy modulation in cancer. Clin Cancer Res. 2009;15(17):5308-16.

64. Doherty J, Baehrecke EH. Life, death and autophagy. Nat Cell Biol. 2018; 20(10):1110-7.

65. Jen J, Wang YC. Zinc finger proteins in cancer progression. J Biomed Sci. 2016;23(1):53

66. Lai KP, Chen J, He M, Ching AK, Lau C, Lai PB, et al. Overexpression of ZFX confers self-renewal and chemoresistance properties in hepatocellular carcinoma. Int J Cancer. 2014;135(8):1790-9.

\section{Publisher's Note}

Springer Nature remains neutral with regard to jurisdictional claims in published maps and institutional affiliations.

Ready to submit your research? Choose BMC and benefit from:

- fast, convenient online submission

- thorough peer review by experienced researchers in your field

- rapid publication on acceptance

- support for research data, including large and complex data types

- gold Open Access which fosters wider collaboration and increased citations

- maximum visibility for your research: over $100 \mathrm{M}$ website views per year

At $\mathrm{BMC}$, research is always in progress.

Learn more biomedcentral.com/submissions 